\title{
A systematic optimization of styrene biosynthesis in Escherichia coli BL21(DE3)
}

\author{
Changqing Liu' ${ }^{1,2}$, Xiao Men ${ }^{1,2}$, Hailin Chen ${ }^{1,2}$, Meijie Li ${ }^{1,2}$, Zhaorui Ding ${ }^{4}$, Guoqiang Chen ${ }^{1,2}$, Fan Wang ${ }^{1,2}$, \\ Haobao Liu ${ }^{3}$, Qian Wang ${ }^{3}$, Youshuang Zhu ${ }^{4}$, Haibo Zhang ${ }^{1,2^{*}}$ and Mo Xian ${ }^{1,2^{*}}$
}

\begin{abstract}
Background: Styrene is a versatile commodity petrochemical used as a monomer building-block for the synthesis of many useful polymers. Although achievements have been made on styrene biosynthesis in microorganisms, several bottleneck problems limit factors for further improvement in styrene production.

Results: A two-step styrene biosynthesis pathway was developed and introduced into Escherichia coli BL21(DE3). Systematic optimization of styrene biosynthesis, such as enzyme screening, codon and plasmid optimization, metabolic flow balance, and in situ fermentation was performed. Candidate isoenzymes of the rate-limiting enzyme phenylalanine ammonia lyase (PAL) were screened from Arabidopsis thaliana (AtPAL2), Fagopyrum tataricum (FtPAL), Petroselinum crispum (PCPAL), and Artemisia annua (AaPAL). After codon optimization, AtPAL2 was found to be the most effective one, and the engineered strain was able to produce $55 \mathrm{mg} / \mathrm{L}$ styrene. Subsequently, plasmid optimization was performed, which improved styrene production to $103 \mathrm{mg} / \mathrm{L}$. In addition, two upstream shikimate pathway genes, aroF and pheA, were overexpressed in the engineered strain, which resulted in styrene production of $210 \mathrm{mg} / \mathrm{L}$. Subsequently, combined overexpression of tktA and ppsA increased styrene production to $275 \mathrm{mg} / \mathrm{L}$. Finally, in situ product removal was used to ease the burden of end-product toxicity. By using isopropyl myristate as a solvent, styrene production reached a final titer of $350 \mathrm{mg} / \mathrm{L}$ after $48 \mathrm{~h}$ of shake-flask fermentation, representing a $636 \%$ improvement, which compared with that achieved in the original strain.
\end{abstract}

Conclusions: This present study achieved the highest titer of de novo production of styrene in E. coli at shake-flask fermentation level. These results obtained provided new insights for the development of microbial production of styrene in a sustainable and environment friendly manner.

Keywords: Styrene, L-phenylalanine, Phenylalanine ammonia lyase, aroF, pheA, tktA, ppsA, In situ product removal, Isopropyl myristate, Escherichia coli

\section{Background}

Biorenewable fuels and chemicals have been receiving more and more attention owing to the increasing depletion of fossil fuel resources and concerns about sustainable development of conventional petrochemical industry. Currently, a number of natural and artificial biochemicals, such as succinic acid [1], lactic acid [2], isoprene [3], $\alpha$-pinene [4], $n$-butanol [5], 2-phenylethanol [6], sabinene

\footnotetext{
*Correspondence: zhanghb@qibebt.ac.cn; xianmo@qibebt.ac.cn ${ }^{1}$ CAS Key Laboratory of Biobased Materials, Qingdao Institute of Bioenergy and Bioprocess Technology, Chinese Academy of Sciences, No.189 Songling Road, Laoshan District, Qingdao 266101, China Full list of author information is available at the end of the article
}

[7], as well as numerous fatty acids [8], and their derivatives [9] can be synthesized from renewable substrates such as glucose by microbes. Along with the development of metabolic engineering and the discovery of new metabolic routes, compounds that were previously unobtainable are becoming available through biosynthesis in microbes.

Styrene is a versatile commodity chemical, with an annual global consumption of approximates 25 million metric tons and occupying an approximately 30 billion USD market [10]. Currently, almost all commercially available styrene is derived from the increasing depletion of fossil fuel resources that are rapidly being depleted. 
Moreover the demand for sustainable resources has impelled the researchers to develop biosynthetic routes for the production of styrene from renewable substrates. Recently, a synthetic biological pathway was employed for production of styrene directly from glucose [11]. The two-step heterologous pathway was designed as follows: $\mathrm{L}$-phenylalanine (L-Phe) was converted to trans-cinnamic acid (ferulic acid, tCA), catalyzed by phenylalanine ammonia lyase (PAL, EC 4.3.1.24). Subsequently, tCA was converted to styrene, catalyzed by ferulic acid decarboxylase (FDC1, EC 4.1.1.102, a phenyl acrylate decarboxylase) (Fig. 1). This pathway was first expressed in Escherichia coli NST74, which was previously developed for L-Phe production and the resulting strain was able to produce $260 \mathrm{mg} / \mathrm{L}$ styrene from $15 \mathrm{~g} / \mathrm{L}$ glucose, with a styrene toxicity threshold of $300 \mathrm{mg} / \mathrm{L}$ [11]. However, low PAL activity acted as the flux limiting condition in the engineered styrene biosynthesis pathway because enough L-Phe titers were detected, while tCA titers were observed to remain low throughout, indicating that almost all of the synthesized tCA could be quickly converted to styrene by overexpressed FDC1 [11]. Moreover, although addition of exogenous L-Phe significantly improved the net production of styrene, this approach towards improving styrene biosynthesis is not sustainable, and endogenous L-Phe production must be enhanced in the host platform [11].
Besides, styrene toxicity was a limiting factor, which must be addressed if bio-styrene becomes an alternative to traditional chemical processes [11].

PAL is the first enzyme that leads the L-Phe metabolism into styrene biosynthesis, and its activity has significant effects on styrene yield. To enhance styrene production, one of the most common strategies is to search for PAL with higher activity by screening new isoenzymes from different sources. Nielsen et al. examined four PAL isoenzymes, from Arabidopsis thaliana (AtPAL1 and AtPAL2), Nostoc puntiformes (NpPAL), and Anabaena variabilis (AvPAL), respectively, for their suitability to produce tCA in E. coli, and found that AtPAL2 had the highest enzyme activity among the four isoenzymes investigated [11]. Moreover, although directed evolution and random mutagenesis have been widely used to enhance enzymatic activity, PAL with higher activity has not yet been reported [12].

Numerous methods to increase endogenous L-Phe have been developed [13-18]. It has been demonstrated that L-Phe exhibits feedback inhibition on some of its key biosynthesis pathway genes, such as aroF and pheA, encoding 3-deoxy-D-arabino-heptulosonate-7-phosphate synthetase (DAHPS), and chorismate mutase-prephenate dehydratase (CM-PDT), respectively. Overexpression or deregulation of these key genes could remarkably

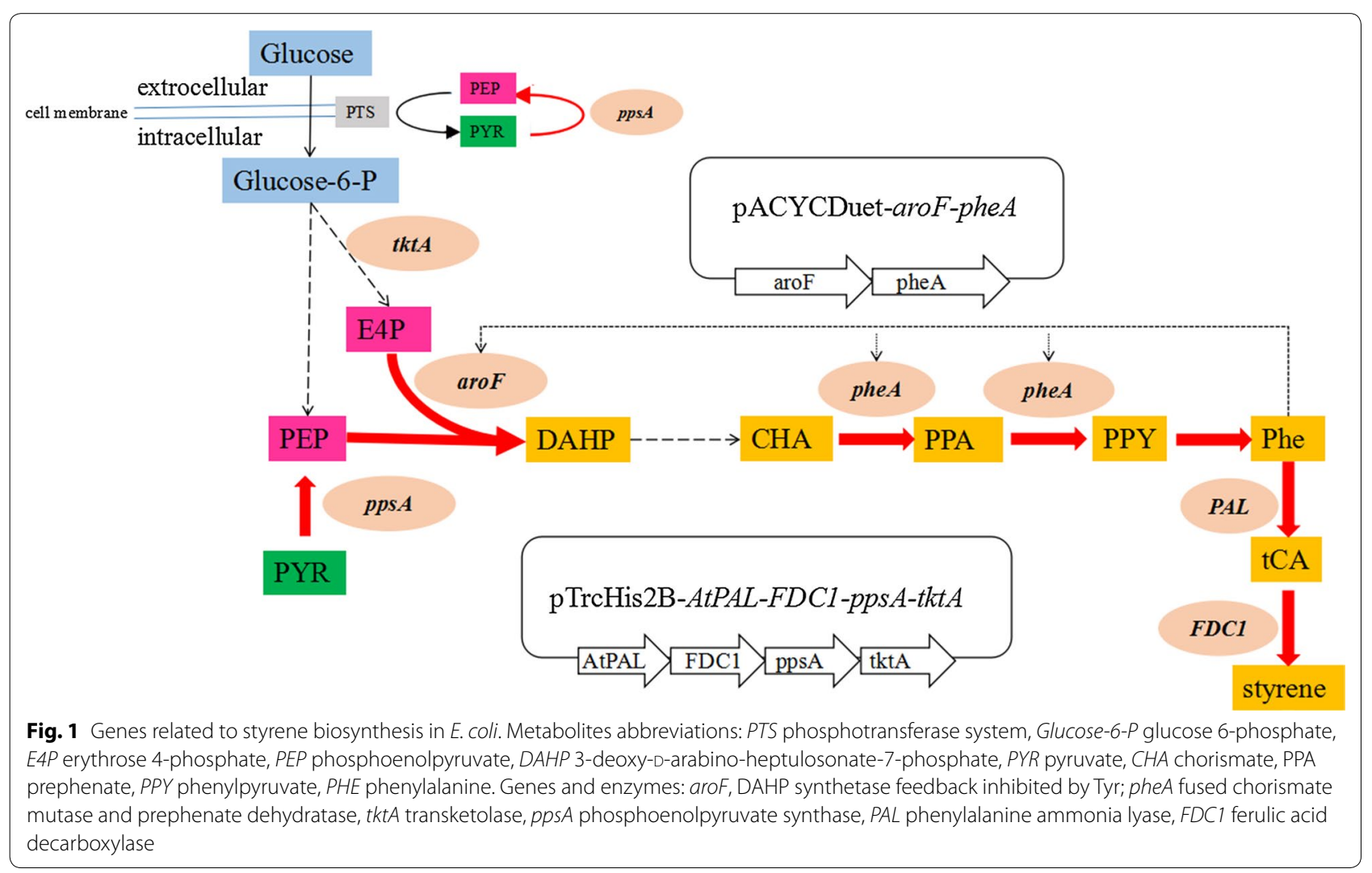


enhance the biosynthesis of the downstream aromatic compounds. In a previous study, zhou et al. co-expressed a feedback-resistant $a r o F^{\mathrm{fbr}}$ and a wild-type $p h e A^{\mathrm{wt}}$ in an L-Tyr auxotrophic E. coli strain WSH-Z06, and increased L-Phe production to $35.38 \mathrm{~g} / \mathrm{L}$, which was 2.81 -fold higher than that achieved in the original strain [19].

Another essential strategy to improve L-Phe production is to enhance the availability of intracellular phosphoenolpyruvate (PEP) and erythrose 4-phosphate (E4P), which are two import precursors involved in L-Phe biosynthesis, from the central metabolic pathway. Overexpression of the key central metabolic pathway genes pps $A$ and $t k t A$ genes is known to increase the availability of PEP and E4P, respectively, and introduction of these genes has been reported to increase aromatic compounds production in engineered E. coli strains [20, 21]. Many biotechnological processes are limited owing to product cytotoxicity or formation of toxic by-products. To avoid these drawbacks, in situ product removal (ISPR) techniques have been developed. This strategy has been demonstrated to be effective in improving the bio-production of some aromatic compounds, such as L-Phe and 2-phenylethanol [22-25].

In the present study, a systematic optimization of styrene biosynthesis in E. coli BL21(DE3) was performed. To obtain PAL with higher activity, candidate isoenzymes from different sources were screened and their encoding genes were optimized based on the preferred codon usage of $E$. coli. Then, the optimized pathway genes were cloned into different plasmids to select for the suitable plasmid that was effective for enzyme expression and styrene production. Subsequently, four upstream pathway genes aroF, pheA, tktA, and ppsA were overexpressed to increase the carbon flow to L-Phe. Finally, to alleviate the toxic effect of endogenous styrene on host cells, ISPR with different solvents on engineered $E$. coli cultures was investigated.

\section{Results and discussion}

\section{Screening PAL isoenzymes and codon optimization}

In a previous study, Nielsen et al. confirmed that PAL was the rate-limiting enzyme in the styrene biosynthesis because the tCA titers remained low throughout [11]. In this study, to screen for a more efficient PAL, three candidate isoenzymes from Petroselinum crispum (PcPAL), Fagopyrum tataricum (FcPAL), and Artemisia annua (AaPAL), were screened with AtPAL2 as a control. It has been reported that recombinant PcPAL exhibits activity towards its natural substrate $\mathrm{L}$-Phe, with a $K m$ value of $116 \pm 4$ and Kcat value of $1 \pm 0.05$, and might have better catalytic properties [26]. Both FtPAL and AaPAL come from medicinal and nutrient-rich plants with high levels of flavonoids, and recombinant FtPAL protein has been found to be specific to L-Phe, with an activity of up to $35.7 \mathrm{IU} / \mathrm{g}[27,28]$. In the present study, to improve expression level, codon-optimized versions of all the above-mentioned genes were synthesized. FDC1 from Saccharomyces cerevisiae and optimized PALs were cloned into the pACYCDuet-1 vector and introduced into $E$. coli $\mathrm{BL} 21$ (DE3), and the obtained transformants were inoculated into M9 medium for $24 \mathrm{~h}$. As shown in Fig. 2a, the expression of FDC1 with AtPAL2 led to production of $55 \mathrm{mg} / \mathrm{L}$ styrene, which was higher than that achieved with FtPAL $(44 \mathrm{mg} / \mathrm{L})$ or PCPAL $(36 \mathrm{mg} / \mathrm{L})$, respectively, whereas the strains containing $A a P A L$ rarely produced styrene. Although no enzyme with higher activity was detected, both FtPAL and PcPAL were confirmed to be specific to L-Phe because gas chromatography-mass spectrometry (GC-MS) did not detect 4-Vinylphenol in the fermentation products (Additional file 1: Figure S1). Based on these results, the expression of AtPAL2 combined with FDC1 was used for the production of styrene in subsequent experiments.

\section{Selection of a suitable plasmid for increasing styrene production}

Production of recombinant proteins in E. coli cells is affected by the number of plasmids, as well as their structural and segregational stability, which have essential impacts on productivity [29]. To achieve a high styrene producing capability, a two-step pathway was incorporated into four plasmids with different copy numbers and different promoters to assess any effect on styrene production. All the strains produced styrene from an initial glucose concentration of $15 \mathrm{~g} / \mathrm{L}$ under aerobic conditions in $600-\mathrm{mL}$ flasks. As shown in Fig. $2 \mathrm{~b}$, the strains with high-copy-number plasmids achieved higher styrene titers. E. coli BL06 harboring high-copy-number plasmid pET-28a-AtPAL2-FDC1 produced $70 \mathrm{mg} / \mathrm{L}$ styrene, which had a 123 and 507\% improvement compared with that achieved in E. coli BL01 harboring the mediumcopy-number plasmid pACYCDuet-AtPAL2-FDC1 and $E$. coli BL07 harboring the low-copy-number plasmid pColADuet-AtPAL2-FDC1, respectively. In contrast, plasmid copy number had little effect on cell growth during the process of styrene fermentation, with $\mathrm{OD}_{600}$ of all strains were around 2.0 (Fig. 2b). In addition to the effect of plasmid copy number, the promoter also had a significant influence on styrene production. E. coli BL05 harboring trc promoter plasmid pTrcHis2B-AtPAL2-FDC1 produced $103 \mathrm{mg} / \mathrm{L}$ styrene under the same conditions, which was a $146 \%$ higher than that produced by $E$. coli BL06 harboring T7 promoter plasmid pET-28a-AtPAL2$F D C 1$. Based on these results, E. coli BL05 harboring plasmid pTrcHis2B-AtPAL2-FDC1 was chosen as the parent strain for further styrene production optimization. 

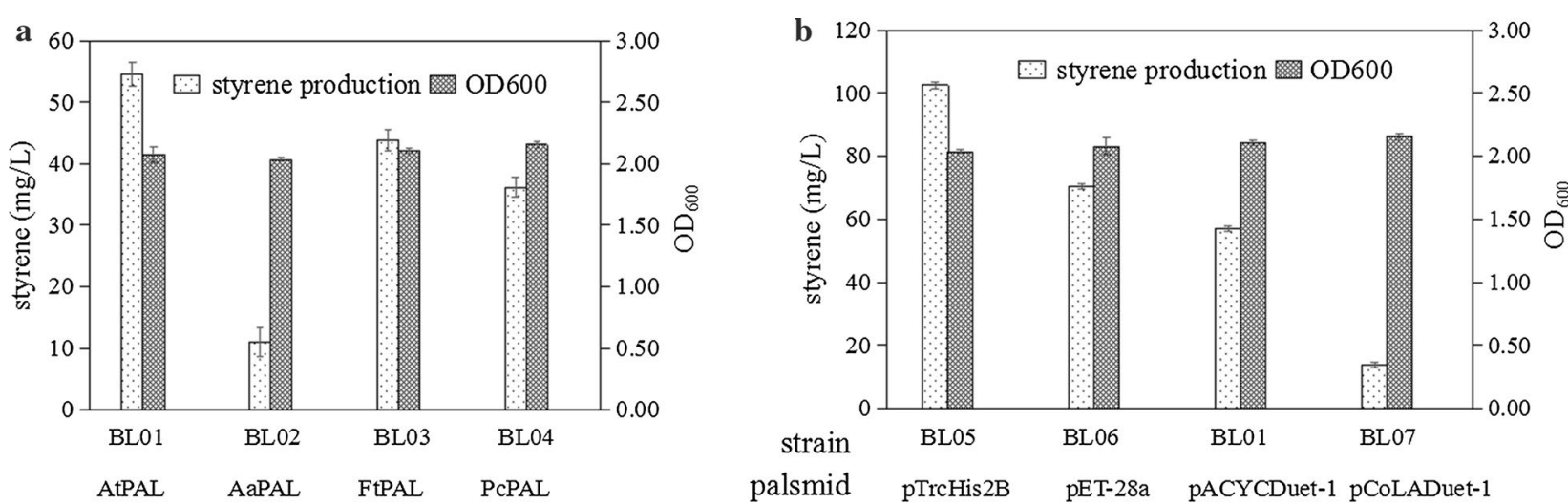

$\begin{array}{ccccc}\text { palsmid } & \text { pTrcHis2B } & \text { pET-28a } & \text { pACYCDuet-1 } & \text { pCoLAD } \\ \text { promoter } & \text { Ttrc } & \text { T7 } & \text { T7 } & \text { T7 } \\ \text { origin } & \text { pBR322 } & \text { pBR322 } & \text { ColA } & \text { P15 } \\ \text { copy level } & \text { high } & \text { high } & \text { medium } & \text { low }\end{array}$

Fig. 2 Effect of PALs and different plasmids on styrene production. a Styrene production with the expression of different PAL candidate genes from P. crispum (PcPAL), F. tataricum (FCPAL), A. annua (AaPAL), and A. thaliana(AtPAL) in recombinant E. coli BL21(DE3). b Styrene production in recombinant E. coli BL21(DE3) harboring different plasmids (pTrCHis2B, pET-28a, pACYCDuet-1, and pColADuet-1) in recombinant E. coli BL21 (DE3). Error bars represent one standard deviation from triplicate experiments

\section{Effect of co-expression of upstream genes aro $F$ and $p h e A$ on styrene production}

Aromatic amino acids such as L-Phe are naturally produced mainly from the shikimate pathway. The first rate-limiting step in this pathway is the condensation reaction between PEP and E4P to form 3-deoxy-D-arabino-heptulosonate 7-phosphate (DAHP). This reaction is catalyzed by three DAHPS isoenzymes encoded by three genes aroF, aroG, and aroH, respectively. The second rate-limiting step is the conversion of chorismate into phenylpyruvate, via prephenate, catalyzed by CM-PDT, which is encoded by pheA. In a previous study, Backman et al. utilized a genetically modified $E$. coli strain with $p h e A^{\mathrm{fbr}}$ and $a r o F^{\mathrm{fbr}}$ genes to improve the metabolic flux towards L-Phe biosynthesis and achieved $50 \mathrm{~g} / \mathrm{L} \mathrm{L}-\mathrm{Phe}$ with a yield of 0.25 (mol L-Phe/mol glucose) [30], which was the highest L-Phe production reported thus far. In our study, to enhance upstream pathway flux, aroF and pheA, were overexpressed in E. coli BL05 and the resultant strain E. coli BL0501 was evaluated for its ability to produce styrene in shake flask. After $24 \mathrm{~h}$ of cultivation, the styrene titers produced by E. coli BL0501 reached $210 \mathrm{mg} / \mathrm{L}$, while the control strain E. coli BL0500 produced $100 \mathrm{mg} / \mathrm{L}$ styrene (Fig. 3a). These results confirmed that aroF and pheA genes are the key factors determining the biosynthesis of endogenous L-Phe and co-expression of CM-PDT and DAHPS could significantly improve L-Phe and L-Phe derivatives produced by E. coli, similar to that reported in previous studies [31, 32].

\section{Effect of co-expression of central metabolic pathway genes} tkt $A$ and $p p s A$ on styrene production

To produce one molecule of $\mathrm{L}$-Phe, two molecules of PEP and one molecule of E4P, which are both involved in the central metabolic pathways, are required. PEP is predominantly utilized in the phosphotransferase system (PTS), which is responsible for the translocation and phosphorylation of glucose, converting one PEP molecule to pyruvate (Fig. 1) [33]. Enhancing the expression level of PEP synthase (encoded by ppsA), which recycles pyruvate generated by PTS-mediated glucose transport to PEP, is an important approach for increasing the carbon flux from PEP to the aromatic amino acids pathway [33]. E4P can be directly produced by transketolase (encoded by $t k t A$ ) or transaldolase (encoded by talB), and $t k t A$ have been demonstrated to be more effective in directing the carbon flux to the aromatic pathway than talB [34].

In our study, to investigate the effect of overexpression of $p p s A$ and $t k t A$ on styrene production, styrene production of engineered $E$. coli strains harboring different constructs in shake-flask fermentation was investigated. As shown in Fig. 3b, E. coli BL0801 (harboring pTrc-AtPAL2-FDC1-ppsA-tktA and pACYC-aroF-pheA) accumulated $275 \mathrm{mg} / \mathrm{L}$ styrene after $24 \mathrm{~h}$ of fermentation with the consumption of $6.7 \mathrm{~g} / \mathrm{L}$ glucose, which represents a 131 and $268 \%$ improvement over styrene production by E. coli BL08 (harboring pTrc-AtPAL2-FDC1 and pACYC-aroF-pheA) and E. coli BL05 (harboring pTrcAtPAL2-FDC1), respectively. When compared with the previous study, which overexpressed AtPAL2 and 

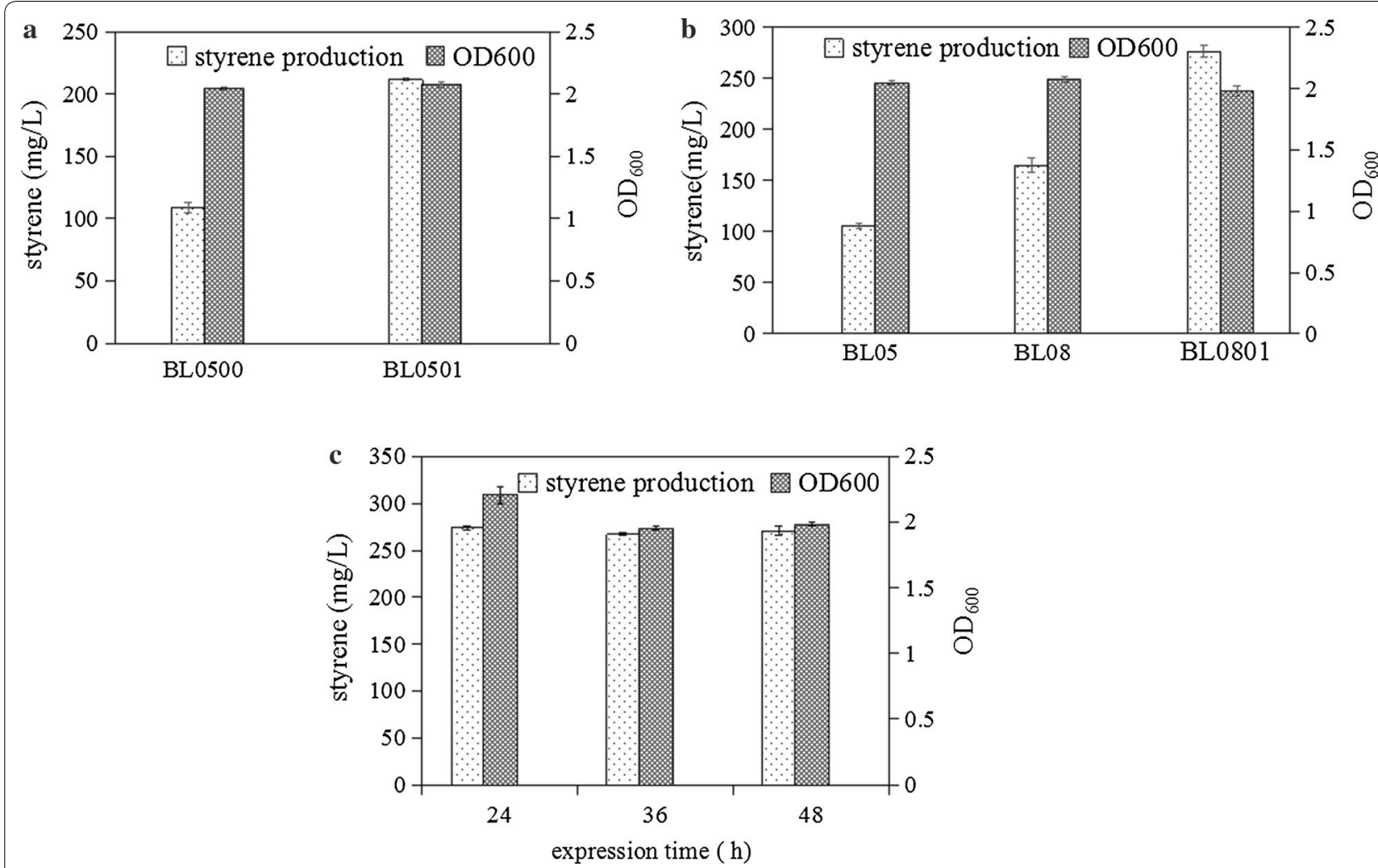

Fig. 3 Effects of overexpression of key upstream genes on styrene production. a Effect of overexpression of aroF and pheA genes on styrene production. E. coli BL0501 and BL0500 cells were cultivated and their cell growth (OD600) and styrene production titers were compared. b Effect of overexpression of aroF, pheA, ppsA, and tktA genes on styrene production. E. coli BL0801, BL08, and BL0500 cells were cultivated and their cell growth (OD600) and styrene production titers were compared. c Optimization of induction length. E. coli BL0801 cells were induced for 24, 36, and $48 \mathrm{~h}$ and their cell growth (OD600) and styrene production titers were compared. Error bars represent one standard deviation from triplicate experiments

FDC1 in an L-Phe overproduction strain E. coli NST74 (aroH367, tyrR366, tna-2, lacY5, aroF394(fbr), malT384, pheA101(fbr), pheO352, aroG397(fbr)) and the resulting strain was able to produce $260 \mathrm{mg} / \mathrm{L}$ styrene from $15 \mathrm{~g} / \mathrm{L}$ glucose [11], it seemed that the styrene titer achieved in E. coli BL0801 was not improved as much as expected. Several reasons might be responsible for this result. Different genetic backgrounds between E. coli NST74 (K-12) and $E$. coli BL21(DE3) may result in different styrene yields. Furthermore, $\operatorname{aro} F^{f b r}$, $\operatorname{aro} G^{\mathrm{fbr}}, t y r R$, $p h e A^{\mathrm{fbr}}$, and pheO were overexpressed in $E$. coli NST74, while $\operatorname{aro} F^{\mathrm{wt}}$, $p h e A^{\mathrm{wt}}, t k t A$, and ppsA were introduced into $E$. coli $\mathrm{BL} 21(\mathrm{DE} 3)$. Multiple isozymes encoding genes, $\operatorname{aro} F$ and aroG, phe A, and pheO were co-expressed, which may significantly increase the flow of central metabolic carbon to phenylalanine biosynthesis. In the case, our engineered strain only had a slightly higher styrene production than the strain using E. coli NST74 as a host. Therefore, different host including E. coli NST74 (K-12) and more genes would be considered to increase the production of styrene in the subsequent research.
During the fermentation, the growth of engineered $E$. coli strains was very slow, with $\mathrm{OD}_{600}$ reaching 2.0 after $24 \mathrm{~h}$ of induction. Therefore, the effect of induction time on styrene production was examined. As shown in Fig. 2c, styrene production and $\mathrm{OD}_{600}$ did not increase along with the elongation of induction time, which could possibly be owing to the inhibition of cell growth by styrene produced by the host strains. This result reconfirmed that product toxicity is a limiting factor that must be addressed in addition to metabolic regulations.

\section{Styrene toxicity assay}

To evaluate styrene toxicity on E. coli BL21(DE3), the effect of exogenous addition of styrene at different concentrations $(100,200,300$, and $400 \mathrm{mg} / \mathrm{L})$ on growing cultures was investigated. As shown in Fig. $4 \mathrm{a}$, an $\mathrm{OD}_{600}$ of 3.4 was reached in the absence of styrene. When the concentration of styrene was less than $300 \mathrm{mg} / \mathrm{L}$, no significant growth inhibition was observed. However, when styrene concentration was increased to more than $300 \mathrm{mg} / \mathrm{L}$, a remarkable cell growth inhibitory effect to 

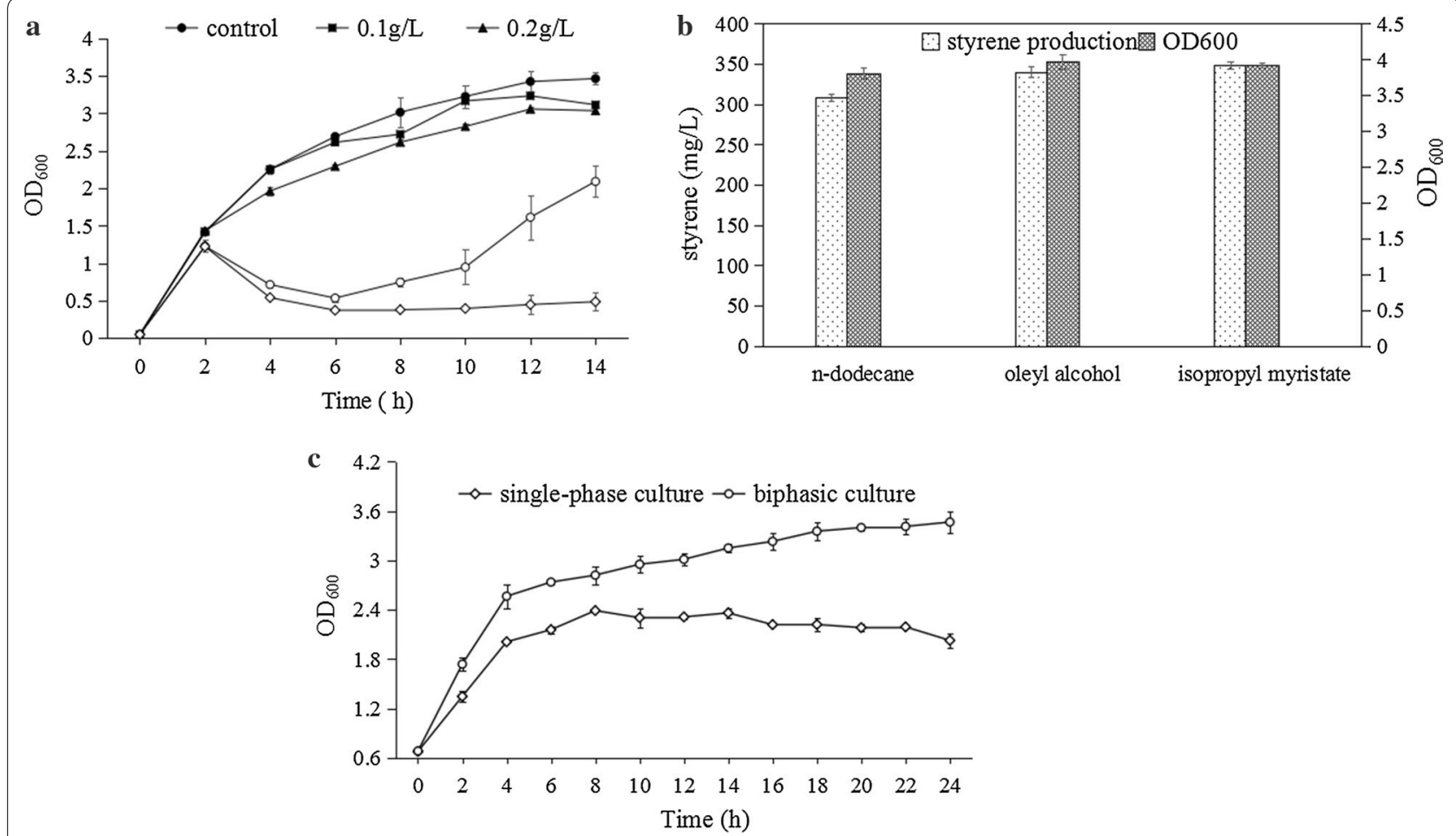

Fig. 4 Tolerance of E. coli BL21(DE3) cells to styrene toxicity and ISPR with different solvents. a Growth response of E. coli BL21(DE3) cells to 0, 100, 200, 300, and $400 \mathrm{mg} / \mathrm{L}$ styrene in LB medium. b Effect of different solvents on styrene production and cell growth. cTime course of biomass by $E$. coli BL0801 cells in biphasic and single-phase cultures. Error bars represent one standard deviation from triplicate experiments

cell growth was detected at $0-10 \mathrm{~h}$. Interestingly, after $10 \mathrm{~h}$, the values of $\mathrm{OD}_{600}$ started to increase, which could possibly be due to the adaptation of cells to cultivation conditions with styrene or evaporation of styrene due to the insolubility of styrene in water. These findings are consistent with the previously reported styrene toxicity thresholds for E. coli NST74 [11].

After systematic optimization, the styrene output achieved $275 \mathrm{mg} / \mathrm{L}$ in the present study, which was close to the inhibitory threshold $(300 \mathrm{mg} / \mathrm{L})$. However, for economically viable and sustainable production of microbial-derived renewable styrene, the styrene titers and productivity must be ultimately improved, in other words, styrene toxicity must be overcome or effectively circumvented [11]. The accumulation of de novo synthesized biofuels or other solvent-like compounds within the cytoplasmic membrane has been shown to disrupt membrane integrity, resulting in the leakage of ions, metabolites, lipids, and proteins, as well as affecting the cells ability to maintain its internal $\mathrm{pH}$ and an appropriate trans-membrane proton gradient [35, 36]. Efforts have been made to improve host strains for better solvent tolerance, including introduction of efflux pumps or transporters, heat shock proteins, membrane modifications, genome engineering, random mutation, adaptive evolution, and approaches that integrated multiple-tolerance strategies [24]. In addition, ISPR and medium supplements can help to ease the burden of end-product toxicity and may be used in combination with genetic approaches. Therefore, in the present study, ISPR was used in combination with genetic approaches to increase styrene tolerance and production capacity of the host strains.

\section{In situ product removal (ISPR) and solvent selection}

For successful application of this approach, the selection of a suitable solvent is important. Ideal solvents should be biocompatible yet non-bioavailable and display high equilibrium partitioning of the target compound over water [37]. Various kinds of solvents, such as oleic acid, oleyl alcohol, miglyol, isopropyl myristate, and polypropylene glycol have been tested for their ability to improve the production of aromatic compounds [23-25, 38]. In our study, the effect of oleyl alcohol, $n$-dodecane, and isopropyl myristate on cell growth and styrene production of the engineered strain E. coli BL0801 was investigated, with no-solvent culture as a control. After $48 \mathrm{~h}$ of shake-flask fermentation, in the presence of $n$-dodecane, oleyl alcohol, and isopropyl myristate, the styrene titers reached 304,340 , and $350 \mathrm{mg} / \mathrm{L}$, representing 110 , 
$124,125 \%$ improvements, respectively, when compared with that achieved in single-phase cultures $(275 \mathrm{mg} / \mathrm{L})$ (Fig. 4b). In addition, the cell growth curves of the E. coli BL0801 in biphasic and in single-phase cultures were also examined. The results revealed that the cell growth was significantly increased in biphasic culture when compared with that in single-phase culture (Fig. 4c), it is presumed that isopropyl myristate could selectively remove the styrene from the reaction system, thereby maintaining the styrene concentration around the cells below the inhibitory threshold, and allowing the strains to continue styrene production, resulting in higher biphasic culture than that in single-phase culture.

Furthermore, maximum theoretical yield coefficients ${ }^{\max } Y_{P h e} / G l c$ were calculated from the known stoichiometry of L-Phe biosynthesis from glucose, in an engineered strain where either the PTS was inactive or PYR was being recycled back to PEP, and the maximum theoretical yield(eng. $\left.{ }^{\max } Y_{\text {Phe }} / G l c\right)$ was $0.55 \mathrm{~g} / \mathrm{g}[20,39,40]$. Moreover, based on the hypothesis that complete conversion of all endogenously produced L-Phe to styrene is possible (e.g., if the pathway engineered in the present study could achieve a particularly high flux), the maximum theoretical yield (eng. ${ }^{\max } Y_{\text {styrene }} / G l c$ ) was calculated to be $0.35 \mathrm{~g} / \mathrm{g}$. According to this value, in single-phase culture, $E$. coli BL0801 strains reached yields $0.041 \mathrm{~g} / \mathrm{g}$, corresponding to $12 \%$ of the ${ }^{\text {eng. }}$ ${ }^{\max } Y_{\text {styrene }} / G l c$, while in biphasic culture, E. coli BL0801 strains reached yields $0.048 \mathrm{~g} / \mathrm{g}$, corresponding to $14 \%$ of the ${ }^{\text {eng. max }} Y_{\text {styrene }} / G l c$. The above data indicate that it is possible to achieve higher yield efficiency. Major challenges may come from low enzymatic activity and flux imbalance.

In the present study, methods to improve activity of PAL, which is a rate-limiting enzyme in the styrene biosynthesis, were first considered. Significant achievements were made by enzyme engineering, such as screening of enzymes with high activity and specificity [11, 41, 42], mutating the coding sequence in the regulatory domain $[12,43,44]$, and family shuffling recombines natural proteins with high sequence identity [45-47]. Currently, these strategies have not yet been applied to attain PAL with higher activity. Feedback inhibition is one of the fundamental mechanisms that regulates the synthesis of amino acids and avoids their excessive accumulation which may cause imbalanced metabolism [48, 49]. To improve the metabolic flux towards L-Phe biosynthesis, overexpression of feedback-resistant $p h e A\left(p h e A^{\mathrm{fbr}}\right)$ and $\operatorname{aroF}\left(\operatorname{aro} F^{\mathrm{fbr}}\right)$ genes may be effective strategies. However, mutant enzymes may decrease thermostability and catalytic efficiency. For example, the use of the $\operatorname{aro} F^{\mathrm{wt}}$ produced much more L-Phe than aro $F^{\mathrm{fbr}}$ (Asn8-Lys) due to the decreasing thermostability of $\operatorname{aro} F^{\mathrm{fbr}}[50,51]$.

To overcome flux imbalances, rational strategies to regulate gene expression were developed, such as application of inducible promoters, use of non-native RNA polymerase [52], the replacement of the ribosome binding site [53], as well as multivariate modular metabolic engineering [54]. Recently, biosensors have been employed to regulate metabolic flux. Biosensor application is an effective strategy that could dynamically detect pathway flux or the levels of pathway intermediates or products and regulators that respond to sensor input and accordingly regulate enzyme expression [55]. In a report, transcription factor (TF)-based sensor, a mutated transcriptional activator NahR from Pseudomonas putida, was used to detect benzoate and 2-hydroxybenzoate accumulation in E. coli [56]. In another report, researchers utilized a lysine riboswitch (RNA-based) to regulate the expression of citrate synthase and control the metabolic flux of the tricarboxylic acid cycle in a lysine-producing strain Corynebacterium glutamicum LP917, which increased the lysine production by 63\% [57]. However, most applications have been limited to natural sensor-regulators [55]. Modular scaffold strategies are also effective approaches to improve metabolic flux. A scaffold protein carrying multiple protein-protein interaction domains is used to co-localize sequential pathway enzymes that have been tagged with peptide ligands specific for the domains on the scaffold [58]. The combined use of these multi-functional enzymes might increase the yield and titer of aromatic compounds from glucose. However, a major drawback of this method is that it often results in decreased activity of one enzyme or both the enzymes [59].

Based on the above reason, our subsequent work would focus on finding feedback-resistant pheA $\left(p h e A^{\mathrm{fbr}}\right)$ and $\operatorname{aro} F\left(\operatorname{aro} F^{\mathrm{fbr}}\right)$ genes with improving thermostability and catalytic efficiency. Furthermore, screening an efficient method to overcome flux imbalances would lay the foundation for industrialized production of styrene.

\section{Conclusion}

In this study, a series of rational and systematic optimizations of styrene biosynthesis was described (Table 1 ). The $P A L$ gene sequences from $A$. thaliana, $P$. crispum, $F$. tataricum, and $A$. annua were collected, optimized, and synthesized; these genes were based on the preferred codon usage of $E$. coli. The engineered strain $E$. coli BL01 produced $55 \mathrm{mg} / \mathrm{L}$ styrene from glucose in shake-flask cultures. Subsequently, the plasmids were optimized by evaluating the effects of copy numbers and promoters on styrene production. E. coli BL05 harboring plasmid pTrc-AtPAL2-FDC1 strain produced $103 \mathrm{mg} / \mathrm{L}$ styrene in shake-flask fermentation, which represented a $187 \%$ increase in styrene production, when compared with that produced by $E$. coli BL01 harboring plasmid pACYC-AtPAL2-FDC1. Then, the upstream shikimate 
Table 1 Results of the systematic optimization of styrene biosynthesis in E. coli BL21 (DE3)

\begin{tabular}{|c|c|c|c|c|c|c|}
\hline Step & Optimization strategy & Strain & Plasmid & $\begin{array}{l}\text { Yield } \\
\text { (mg/L) }\end{array}$ & $\begin{array}{l}\text { Improvement } \\
\text { over previous step (\%) }\end{array}$ & $\begin{array}{l}\text { Improvement } \\
\text { over original step (\%) }\end{array}$ \\
\hline 1 & $\begin{array}{l}\text { Enzyme screening and codon } \\
\text { optimization }\end{array}$ & E. coli BL01 & PACYC-AtPAL-FDC1 & 55 & - & - \\
\hline 2 & Plasmid optimization & E. coli BL05 & pTrc-AtPAL-FDC1 & 103 & 187 & 187 \\
\hline 3 & Co-expression of aroF and pheA & E. coli BL0501 & $\begin{array}{l}\text { pTrc-AtPAL-FDC1 and pACYC- } \\
\text { aroF-pheA }\end{array}$ & 210 & 203 & 382 \\
\hline 4 & $\begin{array}{l}\text { Co-expression of arof, pheA, } \\
\text { pps } A \text {, and tktA }\end{array}$ & E. coli BL0801 & $\begin{array}{l}\text { pTrc-AtPAL-FDC1-ppsA-tktA and } \\
\text { pACYC-aroF-pheA }\end{array}$ & 275 & 131 & 500 \\
\hline 5 & Expression time optimization & E. coli BL0801 & $\begin{array}{l}\text { pTrc-AtPAL-FDC1-ppsA-tktA and } \\
\text { pACYC-aroF-pheA }\end{array}$ & 275 & 100 & 500 \\
\hline 6 & In situ extraction & E. coli BL0801 & $\begin{array}{c}\text { pTrc-AtPAL-FDC1-ppsA-tktA and } \\
\text { pACYC-aroF-pheA-tktA-ppsA }\end{array}$ & 350 & 127 & 636 \\
\hline
\end{tabular}

pathways genes aroF and pheA were overexpressed in $E$. coli BL0501 strain, which led to a final styrene production at titers of $210 \mathrm{mg} / \mathrm{L}$ in shake-flask fermentation, which represents a $203 \%$ improvement in styrene production, when compared with that achieved in E. coli BL05 strain. Moreover, when combined with the overexpression of central metabolic pathway genes $t k t A$ and pps $A$, the engineered $E$. coli BL0801 produced $275 \mathrm{mg} / \mathrm{L}$ styrene. Fermentation time optimization and toxicity assays confirmed that the endogenously synthesized styrene had inhibitory effects on cell growth. To alleviate the toxic effects of styrene on the host cells, ISPR was applied. When isopropyl myristate was used as the solvent, the styrene titers reached $350 \mathrm{mg} / \mathrm{L}$ after $48 \mathrm{~h}$ of shake-flask fermentation, representing 127 and 636\% improvements in styrene production, when compared with that achieved in cultures without solvent $(275 \mathrm{mg} / \mathrm{L})$ and the original E. coli BL01, respectively. To the best of our knowledge, this is the highest styrene titer produced by de novo production of styrene in $E$. coli in shake-flask cultures.

\section{Methods}

\section{Strains and media}

All the strains and plasmids used in this study are listed in Table 2. E. coli DH5a was used as the host for DNA manipulation and E. coli BL21(DE3) was used as the host to express protein and produce styrene. The strains were grown routinely in Luria-Bertani (LB) broth (supplemented with antibiotics if necessary). For evaluating styrene production in shake-flask fermentation, the strains were grown in a modified M9 medium consisting of the following: $\mathrm{Na}_{2} \mathrm{HPO}_{4}, 6 \mathrm{~g} / \mathrm{L} ; \mathrm{KH}_{2} \mathrm{PO}_{4} 3 \mathrm{~g} / \mathrm{L}$; $\mathrm{NaCl}, 0.5 \mathrm{~g} / \mathrm{L} ; \mathrm{NH}_{4} \mathrm{Cl}, 1 \mathrm{~g} / \mathrm{L} ; \mathrm{MgSO}_{4} \cdot 7 \mathrm{H}_{2} \mathrm{O}, 0.492 \mathrm{~g} / \mathrm{L}$; $\mathrm{CaCl}_{2}, 0.11098 \mathrm{~g} / \mathrm{L}$; Thiamine-HCl, $0.01 \mathrm{~g} / \mathrm{L} ;$ glucose, $15 \mathrm{~g} / \mathrm{L}$ and $1 \mathrm{~mL} / \mathrm{L}$ trace element solution which includes $0.37 \mathrm{~g} / \mathrm{L}\left(\mathrm{NH}_{4}\right)_{6} \mathrm{Mo}_{7} \mathrm{O}_{24} \cdot 4 \mathrm{H}_{2} \mathrm{O}, 0.29 \mathrm{~g} / \mathrm{L} \mathrm{ZnSO}_{4} \cdot 7 \mathrm{H}_{2} \mathrm{O}$,
$2.47 \mathrm{~g} / \mathrm{L} \mathrm{H}_{3} \mathrm{BO}_{4}, 0.25 \mathrm{~g} / \mathrm{L} \mathrm{CuSO}_{4} \cdot 5 \mathrm{H}_{2} \mathrm{O}$, and $1.58 \mathrm{~g} / \mathrm{L}$ $\mathrm{MnCl}_{2} \cdot 4 \mathrm{H}_{2} \mathrm{O}$. DNA polymerase and DNA marker were purchased from TransGen Biotech. Restrictions enzymes and the DNA ligase were purchased from Thermo Fisher Scientific.

\section{Construction of plasmids}

All the primers used in this study are listed in Table 3. The primers and optimized genes were synthesized by BGI (Beijing, China). Candidate encoding genes from A. thaliana (Accession No. AEE79055.1), F. tataricum (Accession No. GQ285125.1), P. crispum (Accession No. CAA68938.1), and A. annua (Accession No. AKP55356.1) were optimized and synthesized with pUC57-simple as vector. The FDC1 (Accession No. DAA12368.1) from $S$. cerevisiae encoding tCA decarboxylase was also optimized and cloned into vector pACYCDuet-1 between Nde I and $B g l$ II to generate pACYC-FDC1. The candidate $P A L$ gene fragments were digested with Nco I and Not I, and cloned into the corresponding sites of pACYC$F D C 1$ to create pACYC-AtPAL2-FDC1, pACYC-AaPAL$F D C 1$, pACYC-FtPAL-FDC1, and pACYC-PCPAL-FDC1, respectively. Then, the AtPAL2-FDC1 gene fragment was digested with Nco I and Xho I, and cloned into the corresponding sites of pTrcHis2B, pET-28a, and pColADuet-1 to create pTrc-AtPAL2-FDC1, pET-AtPAL2-FDC1, and pColA-AtPAL2-FDC1, respectively (Table 2). The E. coli BL21(DE3) genomic DNA was used as the template for PCR amplification. The aroF and phe $A$ genes were cloned into pACYCDuet-1 to create pACYC-aroF-pheA. The $t k t A$ and $p p s A$ genes were cloned into pTrc-AtPAL2$F D C 1$ to generate pTrc-AtPAL2-FDC1-ppsA-tktA using NEBuilder HiFi DNA Assembly (New England Biolabs).

\section{Cultivation conditions}

Shake-flask fermentation: Seed culture was prepared by cultivating the strain in $5 \mathrm{~mL}$ of LB medium with 
Table 2 Plasmids and strains used in this study

\begin{tabular}{|c|c|c|}
\hline Name & Relevant characteristics & References \\
\hline \multicolumn{3}{|l|}{ Plasmids } \\
\hline pACYCDuet-1 & $\mathrm{P} 15 \mathrm{~A}$ origin; $\mathrm{Cm}^{\mathrm{R}} ; \mathrm{P}_{\mathrm{T}}$ & Novagen \\
\hline pACYCDuet-AtPAL-FDC1 & P15A origin; $\mathrm{Cm}^{\mathrm{R}} ; \mathrm{P}_{\mathrm{T}}-\mathrm{AtPAL}-\mathrm{FDC} 1$ & This work \\
\hline pACYCDuet-AaPAL-FDC1 & $\mathrm{P} 15 \mathrm{~A}$ origin; $\mathrm{Cm}^{\mathrm{R}} ; \mathrm{P}_{\mathrm{T} 7}-\mathrm{AaPAL}-\mathrm{FDC1}$ & This work \\
\hline pACYCDuet-FtPAL-FDC1 & P15A origin; $\mathrm{Cm}^{\mathrm{R}} ; \mathrm{P}_{\mathrm{T}}-\mathrm{FtPAL}-\mathrm{FDC1}$ & This work \\
\hline pACYCDuet-PCPAL-FDC1 & P15A origin; $\mathrm{Cm}^{\mathrm{R}} ; \mathrm{P}_{\mathrm{T}}-\mathrm{PC} P A L-F D C 1$ & This work \\
\hline pACYCDuet-arof-pheA & $\mathrm{P} 15 \mathrm{~A}$ origin; $\mathrm{Cm}^{\mathrm{R}} ; \mathrm{P}_{\mathrm{T} 7}$-arof-pheA & This work \\
\hline pTrcHis2B & pBR322 origin; $A m p^{R} ; P_{T r c}$ & Novagen \\
\hline pTrcHis2B-AtPAL-FDC1 & pBR322 origin; $A m p^{R} ; P_{T r c}-A t P A L-F D C 1$ & This work \\
\hline pTrcHis2B-AtPAL-FDC1-ppsA-tktA & pBR322 origin; $A m p^{R} ; \mathrm{P}_{T r c}-A t P A L-F D C 1-p p s A-t k t A$ & This work \\
\hline pET-28a & f1 origin; $\mathrm{Kan}^{\mathrm{R}} ; \mathrm{P}_{\mathrm{T} 7}$ & Novagen \\
\hline pET-28a-AtPAL-FDC1 & $\mathrm{f} 1$ origin; $\mathrm{Kan}^{\mathrm{R}} ; \mathrm{P}_{\mathrm{T} 7}-$ AtPAL-FDC1 & This work \\
\hline pColiDuet-1 & ColA origin; $\mathrm{Kan}^{\mathrm{R}} ; \mathrm{P}_{\mathrm{T}}-\mathrm{AtPAL}-\mathrm{FDC1}$ & Novagen \\
\hline pColiDuet-AtPAL-FDC1 & ColA origin; $\operatorname{Kan}^{\mathrm{R}} ; \mathrm{P}_{\mathrm{T} 7}$ & This work \\
\hline \multicolumn{3}{|l|}{ Strains } \\
\hline E. coli BL21(DE3) & E. coli B dcm ompT hsdS $\left(\mathrm{rB}^{-} \mathrm{mB}^{-}\right) \mathrm{gal}$ & Takara \\
\hline E. coli DH5a & deo $R$, recA 1 , endA 1 , hsdR17 $\left(\mathrm{rk}^{-}, \mathrm{mk}^{+}\right)$, phoA, supE44, $\lambda^{-}$, thi $^{-1}$, gyrA96, relA1 & Invitrogen \\
\hline BL01 & E. coli BL21(DE3)-harboring pACYCDuet-AtPAL-FDC1 & This work \\
\hline BL02 & E. coli BL21(DE3)-harboring pACYCDuet-AaPAL-FDC1 & This work \\
\hline BL03 & E. coli BL21(DE3)-harboring pACYCDuet-FtPAL-FDC1 & This work \\
\hline BL04 & E. coli BL21(DE3)-harboring pACYCDuet-PCPAL-FDC1 & This work \\
\hline BL05 & E. coli BL21(DE3)-harboring pTrcHis2B-AtPAL-FDC1 & This work \\
\hline BL06 & E. coli BL21(DE3)-harboring pET-28a-AtPAL-FDC1 & This work \\
\hline BL07 & E. coli BL21(DE3)-harboring pColADuet-AtPAL-FDC1 & This work \\
\hline BL0500 & E. coli BL21(DE3)-harboring pTrcHis2B-AtPAL-FDC1 and pACYCDuet-1 & This work \\
\hline BL0501 & E. coli BL21(DE3)-harboring pTrcHis2B-AtPAL-FDC1 and pACYCDuet-aroF-pheA & This work \\
\hline BL08 & E. coli BL21(DE3)-harboring pTrcHis2B-AtPAL-FDC1 -ppsA-tktA & This work \\
\hline BL0801 & E. coli BL21(DE3)-harboring pTrcHis2B-AtPAL-FDC1-ppsA-tktA and pACYCDuet-aroF-pheA & This work \\
\hline
\end{tabular}

Table 3 Primers used in this study

\begin{tabular}{|c|c|c|}
\hline Primers & Nucleotide sequence $^{a}$ & Description \\
\hline aroF-F & GCggatccTATGCAAAAAGACGCGCTGAAT & Start of aroF; forward primer \\
\hline aroF-R & ATAAGAATgcggecgcTTAAGCCACGCGAGCCGT & End of aroF; reverse primer \\
\hline pheA-F & GAagatctcATGACATCGGAAAACCCGTTAC & Start of pheA; forward primer \\
\hline pheA-R & CCGctcgagTTAGGTTGGATCAACAGGCAC & End of pheA; reverse primer \\
\hline ppsA-F & CGATCGCTGACGTCGGTACCctcgagITAAGGAGGTATATATTAATGTCCAACAATGGCTCGT & Start of ppsA; forward primer \\
\hline ppsA-R & CTTTACGTGAGGACATTAATATATACCTCCTTAAaagcttTTATTTCTTCAGTTCAGCCAGG & End of ppsA; reverse primer \\
\hline tktA-F & TGGCTGAACTGAAGAAATAAaagcttTTAAGGAGGTATATATTAATGTCCTCACGTAAAGAGCTT & Start of tktA; forward primer \\
\hline tktA-R & CTTCTGAGATGAGTTTTTGTtctagaTTACAGCAGTTCTTTTGCTTT & End of tktA; reverse primer \\
\hline
\end{tabular}

a Restriction sites are marked in lower case letters. RBS are underline

appropriate antibiotics at $37{ }^{\circ} \mathrm{C}$ and $250 \mathrm{rpm}$ overnight. Then, $1 \mathrm{~mL}$ of the seed culture was then transferred into $600-\mathrm{mL}$ salt water bottle containing $100 \mathrm{~mL}$ of fermentation medium at $37^{\circ} \mathrm{C}$ and $200 \mathrm{rpm}$. When the cell density reached $\mathrm{OD}_{600}$ of $0.6-0.8$, the cultures were induced with $0.4 \mathrm{mM}$ of isopropyl- $\beta$-D-thiogalactopyranoside (IPTG), with plug added onto the bottle, and then incubated at $30{ }^{\circ} \mathrm{C}$ for an additional $48 \mathrm{~h}$.

In situ extraction of styrene in shake flask: A total of $500 \mu \mathrm{L}$ of the seed culture were inoculated into a $250 \mathrm{~mL}$ shake flask containing $50 \mathrm{~mL}$ of M9 medium and incubated aerobically at $37{ }^{\circ} \mathrm{C}$ and $200 \mathrm{rpm}$. When the cell 
density reached an $\mathrm{OD}_{600}$ of $0.6-0.8,0.4 \mathrm{mM}$ IPTG and $10 \mathrm{~mL}$ of desired solvent were added to the cultures, and then incubated at $30^{\circ} \mathrm{C}$ for an additional $48 \mathrm{~h}$.

\section{Styrene toxicity assay}

A total of $1 \mathrm{~mL}$ of the seed culture was transferred into $600 \mathrm{~mL}$ salt water bottle containing $100 \mathrm{~mL}$ of fermentation medium containing styrene with different concentrations of styrene $(0,100,200,300$, and $400 \mathrm{mg} / \mathrm{L}$, respectively) and cultivated at $30{ }^{\circ} \mathrm{C}$ and $200 \mathrm{rpm}$. The cell growth was determined by $\mathrm{OD}_{600}$ measurements using a UV/Vis spectrophotometer.

\section{Evaluation of styrene production in engineered $E$. coli strains}

After fermentation, the cultures were centrifuged at $12,000 \mathrm{rpm}$ for $1 \mathrm{~min}$, and $50 \mathrm{~mL}$ of the supernatant was extracted with $10 \mathrm{~mL}$ of ethyl acetate. Then, the extract was filtrated with $0.22 \mu \mathrm{m}$ nylon membranes and analyzed by GC-MS to confirm the biosynthesis of styrene. M9 medium without inoculation and treated with the same procedures was used as a control.

GC-MS analysis was performed using Agilent 5975C system [7]. The following conditions were used: $50{ }^{\circ} \mathrm{C}$ for $1 \mathrm{~min}$, which was increased at $20^{\circ} \mathrm{C} / \mathrm{min}$ to $240{ }^{\circ} \mathrm{C}$ and held for $10 \mathrm{~min}$. Peak identification was based on the relative retention time and total ion mass spectral comparison with the external standard. Styrene production was quantified by GC, with following program: $50{ }^{\circ} \mathrm{C}$ for $1 \mathrm{~min}$, which was increased at $20^{\circ} \mathrm{C} / \mathrm{min}$ to $240{ }^{\circ} \mathrm{C}$ and held for $1 \mathrm{~min}$.

\section{Additional file}

Additional file 1: Figure S1. Validation of styrene biosynthesis by engineered E. coli. a Engineered E. coli was cultured in LB medium and detected with GC; $\mathbf{b}$ engineered $E$. coli was cultured in M9 medium and detected with GC-MS.

\begin{abstract}
Abbreviations
A. thaliana: Arabidopsis thaliana; F. tataricum: Fagopyrum tataricum; P. crispum: Petroselinum crispum; A. annua: Artemisia annua; ISPR: in situ product removal; tCA: trans-cinnamic acid; L-Phe: L-phenylalanine; FDC1: ferulic acid decarboxylase; PAL: phenylalanine ammonia lyase; DAHPS: 3-deoxy-D-arabino-heptulosonate-7-phosphate synthetase; CM-PDT: chorismate mutase and prephenate dehydratase; GC-MS: gas chromatography-mass spectrometry; PTS: phosphotransferase system; PEP: phosphoenolpyruvate; E4P: erythrose 4-phosphate; DAHP: 3-deoxy-D-arabino-heptulosonate-7-phosphate.
\end{abstract}

\footnotetext{
Authors' contributions

$M X, H B Z, C Q L$, and XM conceived the original research plans; $M X$ and HBZ supervised the project and experiments; CQL performed most of the experiments; ZRD and GQC participated in shake-flask fermentation; HLC and $M J L$ participated in gene cloning and expression; WF, HBL, QW, and YSZ provided technical assistance to CQL; CQL and XM conceived and wrote the manuscript; MX and HBZ supervised and complemented the writing of the manuscript. All authors read and approved the final manuscript.
}

\section{Author details}

${ }^{1}$ CAS Key Laboratory of Biobased Materials, Qingdao Institute of Bioenergy and Bioprocess Technology, Chinese Academy of Sciences, No.189 Songling Road, Laoshan District, Qingdao 266101, China. ${ }^{2}$ University of Chinese Academy of Sciences, Beijing, China. ${ }^{3}$ Key Laboratory for Tobacco, Gene Resources' Tobacco Research Institute, Chinese Academy of Agricultural Sciences, Qingdao 266101, People's Republic of China. ${ }^{4}$ School of Biological Science, Jining Medical University, Jining 272067, People's Republic of China.

\section{Acknowledgements}

This study was supported by National Natural Science Foundation of China (NSF No. 31400084), Hainan's Key Project of Research and Development Plan (No. ZDYF2017155), and Youth Innovation Promotion Association CAS (No. 2017252).

\section{Competing interests}

The authors declare that they have no competing interests.

\section{Ethics approval and consent to participate}

Not applicable.

\section{Publisher's Note}

Springer Nature remains neutral with regard to jurisdictional claims in published maps and institutional affiliations.

Received: 24 September 2017 Accepted: 10 January 2018

Published online: 25 January 2018

\section{References}

1. Song H, Lee SY. Production of succinic acid by bacterial fermentation. Enzyme Microb Technol. 2006;39:352-61.

2. Abdel-Rahman MA, Tashiro Y, Sonomoto K. Recent advances in lactic acid production by microbial fermentation processes. Biotechnol Adv. 2013;31:877-902.

3. Yang J, Xian M, Su S, Zhao G, Nie Q, Jiang X, Zheng Y, Liu W. Enhancing production of bio-isoprene using hybrid MVA pathway and isoprene synthase in E. coli. PLOS ONE. 2012;7:e33509.

4. Yang J, Nie Q, Meng R, Feng H, Jiang X, Zheng Y, Min L, Zhang H, Mo X. Metabolic engineering of Escherichia coli for the biosynthesis of alphapinene. Biotechnol Biofuels. 2013;6:60-60.

5. Ezeji TC, Qureshi N, Blaschek HP. Bioproduction of butanol from biomass: from genes to bioreactors. Curr Opin Biotechnol. 2007;18:220.

6. Zhang H, Cao M, Jiang X, Zou H, Wang C, Xu X, Xian M. De-novo synthesis of 2-phenylethanol by Enterobacter sp. CGMCC 5087. BMC Biotechnol. 2014;14:30.

7. Zhang H, Liu Q, Cao Y, Feng X, Zheng Y, Zou H, Liu H, Yang J, Xian M. Microbial production of sabinene-a new terpene-based precursor of advanced biofuel. Microb Cell Fact. 2014;13:20.

8. Lu X, Vora H, Khosla C. Overproduction of free fatty acids in E. coli: implications for biodiesel production. Metab Eng. 2008;10:333-9.

9. Steen EJ, Kang YS, Bokinsky G, Hu ZH, Schirmer A, Mcclure A, Cardayre SBD, Keasling JD. Microbial production of fatty-acid-derived fuels and chemicals from plant biomass. Nature. 2010;463:559.

10. Lian J, McKenna R, Rover MR, Nielsen DR, Wen Z, Jarboe LR. Production of biorenewable styrene: utilization of biomass-derived sugars and insights into toxicity. J Ind Microbiol Biotechnol. 2016;43:595-604.

11. McKenna R, Nielsen DR. Styrene biosynthesis from glucose by engineered E. coli. Metab Eng. 2011;13:544-54.

12. Bloom JD, Meyer MM, Meinhold P, Otey CR, MacMillan D, Arnold FH. Evolving strategies for enzyme engineering. Curr Opin Struct Biol. 2005; 15:447-52.

13. Jiang $M$, Zhang $H$. Engineering the shikimate pathway for biosynthesis of molecules with pharmaceutical activities in E. coli. Curr Opin Biotechnol. 2016;42:1-6

14. Liu SP, Zhang L, Mao J, Ding ZY, Shi GY. Metabolic engineering of Escherichia coli for the production of phenylpyruvate derivatives. Metab Eng. 2015;32:55-65. 
15. Doroshenko VG, Livshits VA, Airich LG, Shmagina IS, Savrasova EA, Ovsienko MV, Mashko SV. Metabolic engineering of Escherichia coli for the production of phenylalanine and related compounds. Appl Biochem Microbiol. 2015;51:733-50.

16. Rodriguez A, Martinez JA, Flores N, Escalante A, Gosset G, Bolivar F. Engineering Escherichia coli to overproduce aromatic amino acids and derived compounds. Microb Cell Fact. 2014;13:15.

17. Gosset G. Production of aromatic compounds in bacteria. Curr Opin Biotechnol. 2009;20:651-8.

18. Leuchtenberger W, Huthmacher K, Drauz K. Biotechnological production of amino acids and derivatives: current status and prospects. Appl Microbiol Biotechnol. 2005;69:1-8.

19. Zhou H, Liao X, Wang T, Du G, Chen J. Enhanced L-phenylalanine biosynthesis by co-expression of pheA(fbr) and aroF(wt). Bioresour Technol. 2010;101:4151-6.

20. Baez-Viveros JL, Osuna J, Hernandez-Chavez G, Soberon X, Bolivar F, Gosset G. Metabolic engineering and protein directed evolution increase the yield of L-phenylalanine synthesized from glucose in Escherichia coli. Biotechnol Bioeng. 2004;87:516-24.

21. Barker JL, Frost JW. Microbial synthesis of $p$-hydroxybenzoic acid from glucose. Biotechnol Bioeng. 2001;76:376-90.

22. Takors R. Model-based analysis and optimization of an ISPR approach using reactive extraction for pilot-scale L-phenylalanine production. Biotechnol Prog. 2010;20:57-64.

23. Kim B, Cho BR, Hahn JS. Metabolic engineering of Saccharomyces cerevisiae for the production of 2-phenylethanol via Ehrlich pathway. Biotechnol Bioeng. 2014;111:115-24.

24. Cho C, Choi SY, Luo ZW, Lee SY. Recent advances in microbial production of fuels and chemicals using tools and strategies of systems metabolic engineering. Biotechnol Adv. 2015;33:1455-66.

25. Etschmann M, Schrader J. An aqueous-organic two-phase bioprocess for efficient production of the natural aroma chemicals 2-phenylethanol and 2-phenylethylacetate with yeast. Appl Microbiol Biotechnol. 2006;71:440-3.

26. Dima NA, Filip A, Bencze LC, Oláh M, Sátorhelyi P, Vértessy BG, Poppe L. Expression and purification of recombinant phenylalanine ammonialyase from Petroselinum crispum. Stud Univ Babes-Bolyai Ser Chem. 2016;61:21-34.

27. Li CL, Bai YC, Chen H, Zhao HX, Shao JR, Wu Q. Cloning, characterization and functional analysis of a phenylalanine ammonia-lyase gene (FtPAL) from Fagopyrum tataricum gaertn. Plant Mol Biol Rep. 2012;30:1172-82.

28. Yang S, Roberts MF, Phillipson JD. Methoxylated flavones and coumarins from Artemisia annua. Phytochemistry. 1989;28:1509-11.

29. Skulj M, Okrslar V, Jalen S, Jevsevar S, Slanc P, Strukelj B, Menart V. Improved determination of plasmid copy number using quantitative real-time PCR for monitoring fermentation processes. Microb Cell Fact. 2008;7:6.

30. Backman K, O'connor MJ, Maruya A, Rudd E, Mckay D, Balakrishnan R, Radjai M, Dipasquantonio V, Shoda DD, Hatch R. Genetic engineering of metabolic pathways applied to the production of phenylalanine. Ann N Y Acad Sci. 1990:589:16-24.

31. Konstantinov KB, Nishio N, Seki T, Yoshida T. Physiologically motivated strategies for control of the fed-batch cultivation of recombinant Escherichia coli for phenylalanine production. J Ferment Bioeng. 1991;71:350-5.

32. Zhang C, Zhang J, Kang Z, Du G, Yu X, Wang T, Chen J. Enhanced production of $\mathrm{L}$-phenylalanine in Corynebacterium glutamicum due to the introduction of Escherichia coli wild-type gene aroH. J Ind Microbiol Biotechnol. 2013;40:643-51.

33. Chandran SS, Yi J, Draths K, Daeniken RV, Weber W, Frost J. Phosphoenolpyruvate availability and the biosynthesis of shikimic acid. Biotechnol Prog. 2003;19:808-14.

34. Lu JL, Liao JC. Metabolic engineering and control analysis for production of aromatics: role of transaldolase. Biotechnol Bioeng. 1997;53:132.

35. Weber FJ, Ooijkaas LP, Schemen R, Hartmans S, de Bont J. Adaptation of Pseudomonas putida $\mathrm{S} 12$ to high concentrations of styrene and other organic solvents. Appl Environ Microbiol. 1993;59:3502-4.

36. Ramos JL, Duque E, Gallegos M-T, Godoy P, Ramos-González MI, Rojas A, Terán W, Segura A. Mechanisms of solvent tolerance in Gram-negative bacteria. Annu Rev Microbiol. 2002;56:743-68.
37. Bruce LI, Daugulis AJ. Solvent selection strategies for extractive biocatalysis. Biotechnol Prog. 1991;7:116-24.

38. Serp D, Stockar UV, Marison IW. Enhancement of 2-phenylethanol productivity by Saccharomyces cerevisiae in two-phase fed-batch fermentations using solvent immobilization. Biotechnol Bioeng. 2003;82:103-10.

39. Patnaik P, Liao JC. Engineering of Escherichia coli central metabolism for aromatic metabolite production with near theoretical yield. Appl Environ Microbiol. 1994;60:3903-8.

40. Förberg C, Eliaeson T, Häggström L. Correlation of theoretical and experimental yields of phenylalanine from non-growing cells of a rec Escherichia coli strain. J Biotechnol. 1988:7:319-31.

41. LiY, Niu D, Liang Z, Wang Z, Shi G. Purification, characterization and cloning of a thermotolerant isoamylase produced from Bacillus sp. CICIM 304. J Ind Microbiol Biotechnol. 2013;40:437-46.

42. Guo ZP, Qiu CY, Zhang L, Ding ZY, Wang ZX, Shi GY. Expression of aspartic protease from Neurospora crassa in industrial ethanol-producing yeast and its application in ethanol production. Enzyme Microb Technol. 2011:48:148-54.

43. Helmstaedt K, Strittmatter A, Lipscomb WN, Braus GH. Evolution of 3-deoxy-D-arabino-heptulosonate-7-phosphate synthase-encoding genes in the yeast Saccharomyces cerevisiae. Proc Natl Acad Sci USA. 2005:102:9784-9.

44. Hartmann M, Schneider TR, Pfeil A, Heinrich G, Lipscomb WN, Braus GH. Evolution of feedback-inhibited $\beta /$ a barrel isoenzymes by gene duplication and a single mutation. Proc Natl Acad Sci USA. 2003;100:862-7.

45. Furukawa K, Suenaga H, Goto M. Biphenyl dioxygenases: functional versatilities and directed evolution. J Bacteriol. 2004;186:5189.

46. Aharoni A, Gaidukov L, Yagur S, Toker L, Silman I, Tawfik DS. Directed evolution of mammalian paraoxonases PON1 and PON3 for bacterial expression and catalytic specialization. Proc Natl Acad Sci USA. 2004;101:482.

47. Newman LM, Garcia H, Hudlicky T, Selifonov SA. Directed evolution of the dioxygenase complex for the synthesis of furanone flavor compounds. Tetrahedron. 2004:60:729-34.

48. Gu P, Su T, Qi Q. Novel technologies provide more engineering strategies for amino acid-producing microorganisms. Appl Microbiol Biotechnol. 2016;100:2097-105.

49. Tyo KEJ, Ajikumar PK, Stephanopoulos G. Stabilized gene duplication enables long-term selection-free heterologous pathway expression. Nat Biotechnol. 2009;27:760.

50. Gerigk M, Bujnicki R, Ganponkwenkwa E, Bongaerts J, Sprenger G Takors R. Process control for enhanced L-phenylalanine production using different recombinant Escherichia coli strains. Biotechnol Bioeng. 2010;80:746-54.

51. Jossek R, Bongaerts J, Sprenger GA. Characterization of a new feedbackresistant 3-deoxy-D-arabino-heptulosonate-7-phosphate synthase AroF of Escherichia coli. FEMS Microbiol Lett. 2001;202:145-8.

52. Mutka SC, Carney JR, Yaoquan Liu A, Kennedy J. Heterologous production of epothilone C and D in Escherichia coli. Biochemistry. 2006;45:1321-30

53. Salis HM, Mirsky EA, Voigt CA. Automated design of synthetic ribosome binding sites to control protein expression. Nat Biotechnol. 2009;27:946.

54. Yadav VG, De MM, Lim CG, Ajikumar PK, Stephanopoulos G. The future of metabolic engineering and synthetic biology: towards a systematic practice. Metab Eng. 2012;14:233-41.

55. Dahl RH, Zhang F, Alonso-Gutierrez J, Baidoo E, Batth TS, Redding-Johanson AM, Petzold CJ, Mukhopadhyay A, Lee TS, Adams PD, Keasling JD. Engineering dynamic pathway regulation using stress-response promoters. Nat Biotechnol. 2013:31:1039-46.

56. Van SFS, van Beilen JB, Witholt B. Selection of biocatalysts for chemical synthesis. Proc Natl Acad Sci USA. 2006;103:1693-8.

57. Zhou LB, Zeng AP. Exploring lysine riboswitch for metabolic flux control and improvement of L-lysine synthesis in Corynebacterium glutamicum. ACS Synth Biol. 2015;4:729-34.

58. Lee H, DeLoache WC, Dueber JE. Spatial organization of enzymes for metabolic engineering. Metab Eng. 2012;14:242-51.

59. Dueber JE, Wu GC, Malmirchegini GR, Moon TS, Petzold CJ, Ullal AV, Prather KL, Keasling JD. Synthetic protein scaffolds provide modular control over metabolic flux. Nat Biotechnol. 2009;27:753-9. 